\title{
Selenoprotein $P$ as a diabetes-associated hepatokine that impairs angiogenesis by inducing VEGF resistance in vascular endothelial cells
}

\author{
Kazuhide Ishikura • Hirofumi Misu - Masafumi Kumazaki • Hiroaki Takayama • \\ Naoto Matsuzawa-Nagata • Natsumi Tajima • Keita Chikamoto • Fei Lan • Hitoshi Ando • \\ Tsuguhito Ota • Masaru Sakurai • Yumie Takeshita • Kenichiro Kato • Akio Fujimura • \\ Ken-ichi Miyamoto • Yoshiro Saito • Satomi Kameo • Yasuo Okamoto • Yoh Takuwa • \\ Kazuhiko Takahashi • Hiroyasu Kidoya • Nobuyuki Takakura • Shuichi Kaneko • \\ Toshinari Takamura
}

Received: 10 January 2014 / Accepted: 30 May 2014 /Published online: 3 July 2014

(C) Springer-Verlag Berlin Heidelberg 2014

\begin{abstract}
Aims/hypothesis Impaired angiogenesis induced by vascular endothelial growth factor (VEGF) resistance is a hallmark of vascular complications in type 2 diabetes; however, its molecular mechanism is not fully understood. We have previously identified selenoprotein $\mathrm{P}$ ( $\mathrm{SeP}$, encoded by the SEPP1 gene in humans) as a liver-derived secretory protein that induces insulin resistance. Levels of serum SeP and hepatic expression of SEPP1 are elevated in type 2 diabetes. Here, we
\end{abstract}

Kazuhide Ishikura, Hirofumi Misu and Masafumi Kumazaki contributed equally to this work.

Electronic supplementary material The online version of this article (doi:10.1007/s00125-014-3306-9) contains peer-reviewed but unedited supplementary material, which is available to authorised users.

K. Ishikura $\cdot$ H. Misu $\cdot$ M. Kumazaki $\cdot$ H. Takayama $\cdot$

N. Matsuzawa-Nagata $\cdot \mathrm{N}$. Tajima $\cdot \mathrm{K}$. Chikamoto $\cdot$ F. Lan $\cdot$

H. Ando $\cdot$ T. Ota $\cdot$ M. Sakurai $\cdot$ Y. Takeshita $\cdot$ K. Kato $\cdot$ S. Kaneko $\cdot$

T. Takamura $(\bowtie)$

Department of Disease Control and Homeostasis, Kanazawa

University Graduate School of Medical Sciences, 13-1

Takara-machi, Kanazawa, Ishikawa 920-8641, Japan

e-mail: ttakamura@m-kanazawa.jp

M. Kumazaki $\cdot$ H. Ando $\cdot$ A. Fujimura

Division of Clinical Pharmacology, Department of Pharmacology,

Jichi Medical University, Tochigi, Japan

N. Matsuzawa-Nagata $\cdot$ K. Miyamoto

Department of Medicinal Informatics, Kanazawa University

Graduate School of Medical Sciences, Kanazawa, Ishikawa, Japan

M. Sakurai

Department of Epidemiology and Public Health, Kanazawa Medical

University, Uchinada, Japan investigated the effects of SeP on VEGF signalling and angiogenesis.

Methods We assessed the action of glucose on Sepp1 expression in cultured hepatocytes. We examined the actions of SeP on VEGF signalling and VEGF-induced angiogenesis in HUVECs. We assessed wound healing in mice with hepatic SeP overexpression or SeP deletion. The blood flow recovery after ischaemia was also examined by using hindlimb ischaemia model with Sepp1-heterozygous-knockout mice.

Y. Saito

Department of Medical Life Systems, Faculty of Medical and Life

Sciences, Doshisha University, Kyotanabe, Kyoto, Japan

S. Kameo

Department of Public Health, Gunma University Graduate School of

Medicine, Gunma, Japan

Y. Okamoto · Y. Takuwa

Department of Physiology, Kanazawa University Graduate School of

Medical Sciences, Kanazawa, Ishikawa, Japan

K. Takahashi

Department of Nutritional Biochemistry, Hokkaido Pharmaceutical

University, Otaru, Hokkaido, Japan

H. Kidoya $\cdot$ N. Takakura

Department of Signal Transduction, Research Institute for Microbial Diseases, Osaka University, Osaka, Japan 
Results Treatment with glucose increased gene expression and transcriptional activity for Seppl in H4IIEC hepatocytes. Physiological concentrations of SeP inhibited VEGFstimulated cell proliferation, tubule formation and migration in HUVECs. SeP suppressed VEGF-induced reactive oxygen species (ROS) generation and phosphorylation of VEGF receptor 2 (VEGFR2) and extracellular signal-regulated kinase $1 / 2($ ERK1/2) in HUVECs. Wound closure was impaired in the mice overexpressing Sepp1, whereas it was improved in $\mathrm{SeP}^{-1-}$ mice. $\mathrm{SeP} \mathrm{P}^{+/-}$mice showed an increase in blood flow recovery and vascular endothelial cells after hindlimb ischaemia.

Conclusions/interpretation The hepatokine SeP may be a novel therapeutic target for impaired angiogenesis in type 2 diabetes.

Keywords Angiogenesis $\cdot$ Hepatokine $\cdot$ ROS $\cdot$ Selenoprotein $\mathrm{P} \cdot \mathrm{VEGF}$

$\begin{array}{ll}\text { Abbreviations } \\ \text { BSO } & \text { Buthionine sulfoximine } \\ \text { DCF } & 2^{\prime}, 7^{\prime} \text {-Dichlorofluorescein diacetate } \\ \text { ERK1/2 } & \text { Extracellular signal-regulated kinase } 1 / 2 \\ \text { MAPK } & \text { Mitogen-activated protein kinase } \\ \text { ROS } & \text { Reactive oxygen species } \\ \text { SeP } & \text { Selenoprotein P } \\ \text { VEGF(R) } & \text { Vascular endothelial growth factor (receptor) }\end{array}$

\section{Introduction}

Type 2 diabetes is a chronic hyperglycaemic condition that causes various vascular complications, including damage to: small blood vessels, resulting in retinopathy, nephropathy and neuropathy; and large blood vessels, resulting in cardiovascular diseases. Earlier improved glycaemic control is associated with reduced risk for cardiovascular disease in people with type 2 diabetes [1]. However, more recent clinical trials have indicated that strict glycaemic control does not necessarily prevent vascular complications [2]. Hence, beyond glycaemic control, novel therapies to directly treat vascular disease are needed to improve the prognosis of people with type 2 diabetes.

Angiogenesis is a physiological process involving the growth of new blood vessels from pre-existing vascular structures and the subsequent formation of a vascular network. A number of abnormalities associated with angiogenesis have been observed in people with type 2 diabetes [3], and impaired angiogenesis is linked to the development of various vascular complications in diabetes mellitus. Compared with control individuals without diabetes, people with type 2 diabetes show poor development of coronary collateral vessels on coronary angiography [4]. Moreover, a previous study using autopsied hearts reported that people with diabetes have significantly lower capillary densities in areas of myocardial infarction [5]. These reports suggest that the angiogenic response to infarction and/or ischaemia is inhibited at the levels of capillaries and small arterioles in type 2 diabetes. Inadequate vascular formation could attenuate perfusion recovery in response to ischaemia, thereby partially accounting for the poor clinical outcomes in type 2 diabetic patients with coronary heart disease or peripheral artery disease [6, 7]. In addition, insufficient angiogenesis is involved in abnormal wound healing and the development of diabetic skin ulcers [8].

Vascular endothelial growth factor (VEGF) is a major mediator of angiogenesis under physiological and pathophysiological conditions. VEGF binds and phosphorylates its receptors, leading to the activation of a variety of signalling cascades such as the mitogen-activated protein kinase (MAPK) and Akt cascades. Angiogenic gene therapy using plasmids encoding VEGF has been attempted in patients with coronary or peripheral artery diseases [9]. However, diabetes mellitus people often show a poor response to therapeutic angiogenesis [10]. Therefore, VEGF resistance, a defect of VEGF-related signal transduction, has been postulated as a molecular basis for the dysregulated angiogenesis in diabetes mellitus [3, 11]. The molecular mechanisms underlying VEGF resistance in diabetes mellitus are not fully understood.

Selenoprotein P (SeP, encoded by SEPP1 in humans and Seppl in mice) is a secretory protein produced primarily in the liver $[12,13]$. It contains ten selenocysteine residues and functions as a selenium supply protein [14]. We have previously reported that levels of serum SeP and hepatic gene expression of SEPP1 are elevated in type 2 diabetes [15]. More recently, Yang et al have reported that serum levels of $\mathrm{SeP}$ are increased in people with impaired glucose tolerance [16]. SeP impairs insulin signal transduction and induces dysregulation of glucose metabolism in skeletal muscle and liver, indicating that $\mathrm{SeP}$ functions as a type 2 diabetesassociated hepatokine that causes insulin resistance and hyperglycaemia [15]. SeP has heparin-binding properties [17] and is associated with endothelial cells in rat tissues [18], suggesting that SeP exerts some actions on vascular endothelial cells. A previous study using in vitro techniques reported that $\mathrm{SeP}$ has an antioxidative action in vascular endothelial cells [19]. Nevertheless, it is unknown whether SeP plays a role in the angiogenic response.

We speculated that the liver-derived secretory protein SeP contributes to angiogenesis-associated vascular complications in type 2 diabetes by acting directly on vascular endothelial cells. In the current study, we investigated the effects of $\mathrm{SeP}$ on angiogenesis in normal conditions, independently of diabetes, using purified SeP protein and Seppl-deficient mice without the induction of diabetes. 


\section{Methods}

Cell culture HUVECs were cultured in HuMedia EG2 (Kurabo, Osaka, Japan). H4-II-E-C3 cells were cultured in 10\% (vol./vol.) fetal bovine serum (FBS)/DMEM (Gibco, Carlsbad, CA, USA) as previously described [20]. All cellular experiments were approved by the Committee for Cellular Study at our Institute.

Animals The Seppl-deleted mice were produced by homologous recombination with genomic DNA cloned from a Sv129 P1 library [21]. All animal studies were approved by the Committee for Animal Studies at our Institute. See the electronic supplementary material (ESM) for further details.

Measurement of selenium Total selenium concentrations were determined using a modification of Watkinson's method [22, 23]. See the ESM for further details.

SEPP1 promoter assay The human SEPP1 promoter region was cloned to a luciferase reporter vector, and luciferase activities were measured using the dual luciferase assay system (Promega, Madison, WI, USA) [20]. See the ESM for further details.

Cell proliferation assay HUVECs were quantified using Cell Counting Kit-8 (Wako, Osaka, Japan). See the ESM for further details.

Migration assay HUVECs were seeded in the upper chamber of polycarbonate filters, and the number of cells migrating across the filter was counted. See the ESM for further details.

Cell tubule formation assay HUVECs were seeded on plates coated with ECMatrix gel. Endothelial tubule formation was photographed under a microscope. See the ESM for further details.

Matrigel plug implantation assay This assay was performed using a directed in vivo angiogenesis assay inhibition kit (Trevigen, Gaithersburg, MD, USA). See the ESM for further details.

Western blot analysis HUVECs were pretreated with SeP for $24 \mathrm{~h}$. After $2 \mathrm{~h}$ of starvation, HUVECs were stimulated with VEGF for $15 \mathrm{~min}$. See the ESM for further details.

$R N A$ preparation and quantitative real-time Real-time PCR was performed on an ABI-Prism 7900HT (Applied Biosystems, Carlsbad, CA, USA). See the ESM for further details.

Reactive oxygen species generation Intracellular reactive oxygen species (ROS) levels were measured using 2',7'- dichlorofluorescein diacetate (DCF) and quantified using a fluorescent plate reader (Fluoroskan Ascent FL, Yokohama, Japan). See the ESM for further details.

Purification of $\mathrm{SeP} \mathrm{SeP}$ was purified from human plasma using conventional chromatographic methods [14, 24]. See the ESM for further details.

Preparation of human SEPP1 plasmids and overexpression of SeP in mice The human SEPP1 expression plasmids were provided by Kaketsuken (The Chemo-Sero-Therapeutic Research Institute, Tokyo, Japan). Plasmid was injected into the tail vein of mice. See the ESM for further details.

Measurement of serum human SeP in mice injected with human SEPP1 plasmid Serum levels of human SeP were measured by enzyme-linked immunosorbent assays using two monoclonal antibodies [15, 25].

Mouse wound healing model Full-thickness wound was created, and the extent of wound closure was examined. See the ESM for further details.

Hindlimb ischaemia model Mice underwent ligation and segmental resection of the left femoral vessel [26]. See the ESM for further details.

Identification of $\mathrm{CD} 1^{+}$vessels An antibody to CD31 was used for immunostaining. See the ESM for further details.

Calculations and statistical analysis All data were analysed using SPSS version 11.0 (Japanese Windows Edition; SPSS, www.ibm.com/software/analytics/spss/). See the ESM for further details.

\section{Results}

Glucose increases gene expression and transcriptional activity for SeP in cultured hepatocytes To confirm the elevation of $\mathrm{SeP}$ in the livers of people and animal models with type 2 diabetes [15], we examined the action of glucose on Seppl expression in H4-II-EC hepatocytes (Fig. 1). Sepp1 mRNA expression was significantly increased by $25 \mathrm{mmol} / 1$ glucose in a time-dependent manner (Fig. 1a). Additionally, SEPP1 promoter activity as measured by luciferase activity was increased by $25 \mathrm{mmol} / 1$ glucose compared with mannitol (Fig. 1b). These results are consistent with our previous findings showing that treatment with high glucose increases protein levels of SeP in mouse primary hepatocytes [15]. These results indicate that high concentrations of glucose increase the transcriptional activity of $\mathrm{SeP}$ genes in the cultured hepatocytes. 
a

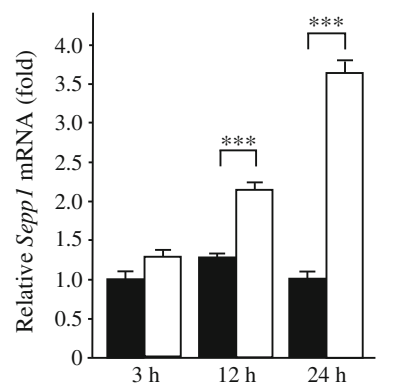

b

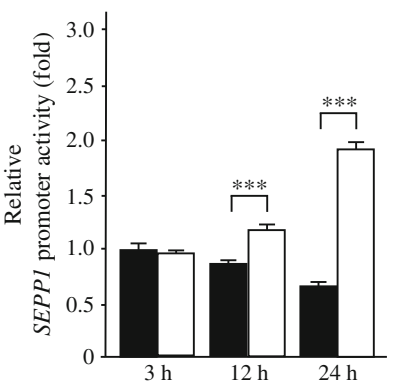

Fig. 1 Glucose increases gene expression and transcriptional activity for $\mathrm{SeP}$ in H4-II-EC3 hepatocytes. (a) Relative Sepp1 mRNA expression normalised to $\beta$-actin. (b) Promoter activity for SEPP1 in H4-II-EC3 hepatocytes treated with glucose and mannitol. Data are mean $\pm \mathrm{SD}, n=3$, $* * * p<0.001$. White bars, glucose; black bars, mannitol

SeP impairs VEGF-induced angiogenesis in endothelial cells To assess the direct action of the liver-derived secretory protein $\mathrm{SeP}$ on vascular endothelial cells, we treated HUVECs with purified human SeP protein. HUVECs were treated with 5 or $10 \mu \mathrm{g} / \mathrm{ml}$ purified human SeP protein, corresponding to serum levels of $\mathrm{SeP}$ in healthy individuals or people with type 2 diabetes [15]. In addition, we confirmed that levels of selenium were undetectable (less than $2.5 \mathrm{ng} / \mathrm{ml}$ ) in all the culture media used for HUVECs. VEGF-induced proliferation of HUVECs was significantly suppressed by treatment with $10 \mu \mathrm{g} / \mathrm{ml} \mathrm{SeP}$ (Fig. 2a). Co-administration of buthionine sulfoximine (BSO), an inhibitor of glutathione synthesis, partly rescued the suppressive effect of SeP.

Next, we examined the effects of SeP on VEGF-induced migration in HUVECs. VEGF promoted the migration of HUVECs across polycarbonate filters. This migration was inhibited by the addition of $\mathrm{SeP}$ in a concentrationdependent manner (Fig. 2b, c). In the absence of VEGF, treatment with SeP did not affect the migration of HUVECs, suggesting that $\mathrm{SeP}$ modulates VEGF-dependent migration of endothelial cells. We further examined the effects of SeP on tubule formation in HUVECs. HUVECs cultured on Matrigel containing VEGF showed morphological tubule formation, with a lumen surrounded by endothelial cells adhering to one another (Fig. 2d). SeP inhibited tubule formation of HUVECs in a concentration-dependent manner (Fig. 2d-e). These in vitro results indicate that $\mathrm{SeP}$ at physiological concentrations impairs VEGF-dependent angiogenesis of vascular endothelial cells.

SeP reduces VEGF-stimulated formation of new vessels in Matrigel The role of SeP in angiogenesis in vivo was further determined by Matrigel plug implantation assay. Matrigel was mixed with VEGF in the presence or absence of SeP protein and the plugs were implanted into the dorsal subcutaneous tissue of mice. After 10 days, angiogenesis inside the Matrigel was quantified. SeP markedly inhibited VEGF-stimulated

$\mathbf{a}$

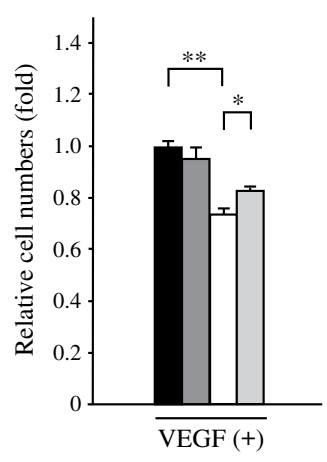

c

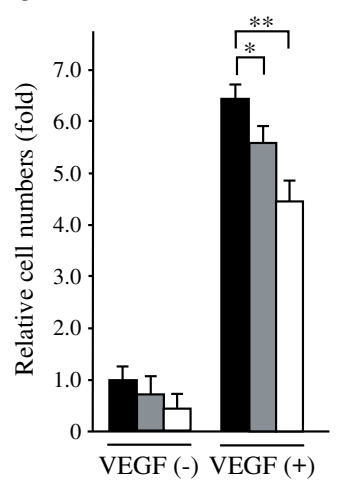

e

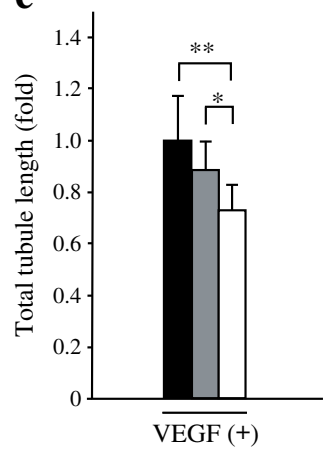

b

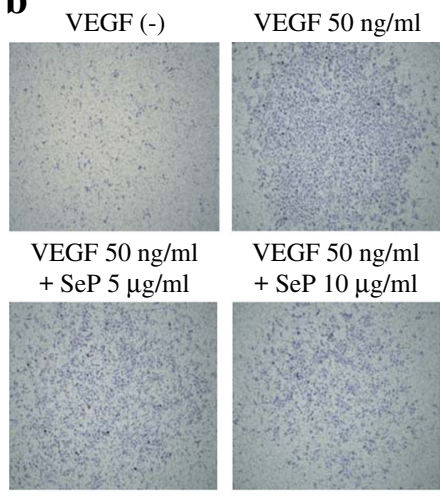

d

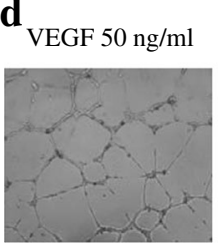

VEGF $50 \mathrm{ng} / \mathrm{ml}$ $+\mathrm{SeP} 5 \mu \mathrm{g} / \mathrm{ml}$

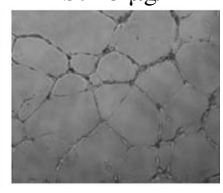

VEGF $50 \mathrm{ng} / \mathrm{ml}$

$+\mathrm{SeP} 10 \mu \mathrm{g} / \mathrm{ml}$

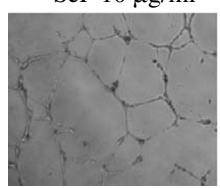

$\mathbf{f}$

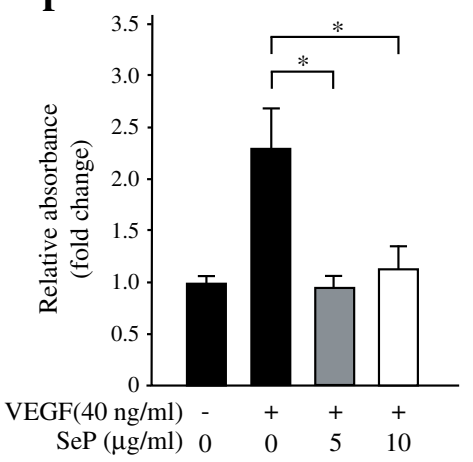

Fig. 2 SeP suppresses VEGF-stimulated angiogenesis in vascular endothelial cells. (a) Cell proliferation in HUVECs treated with VEGF for $48 \mathrm{~h}$ $(n=12)$. (b) Representative images of HUVECs that migrated across the polycarbonate filters (magnification $\times 200$ ). (c) Quantification of HUVECs that migrated across the filters $(n=8)$. (d) Representative images of HUVECs that were subjected to Matrigel tubule formation assay (magnification $\times 400)$. (e) Quantification of total tubule length of HUVECs $(n=9)$. (f) Matrigel implant assay in mice $(n=6-8)$. Data are mean \pm SEM, $* p<0.05$ and $* * p<0.01$. Black bars, control; dark-grey bars, SeP $5 \mu \mathrm{g} / \mathrm{ml}$; white bars, SeP $10 \mu \mathrm{g} / \mathrm{ml}$; light-grey bars, SeP $10 \mu \mathrm{g} / \mathrm{ml}$ and BSO $0.2 \mathrm{mmol} / \mathrm{l}$

formation of new vessels in the Matrigel (Fig. 2f). These results further indicate that $\mathrm{SeP}$ impairs angiogenesis in vivo.

SeP impairs VEGF signal transduction in endothelial cells Next, we determined whether SeP affects VEGF signal transduction in endothelial cells. Pretreatment with SeP 
impaired VEGF-stimulated phosphorylation of VEGF receptor (VEGFR)2 (Tyr1175) and extracellular signal-regulated kinase 1/2 (ERK1/2) (Thr202/Tyr204) in HUVECs (Fig. 3a, b). Co-administration of BSO partially rescued the inhibitory effect of SeP on VEGF signalling (Fig. 3a, b). The mRNA expression of VEGFR2 (also known as KDR) in HUVECs was unaffected by treatment with purified human SeP protein (Fig. 3c). These results indicate that SeP at physiological concentrations impairs VEGF signal transduction in vascular endothelial cells.

SeP suppresses VEGF-induced acute generation of ROS in HUVECS To clarify the mechanism by which the antioxidative protein SeP impairs VEGF signalling, we assessed the action of $\mathrm{SeP}$ on the acute generation of ROS stimulated by
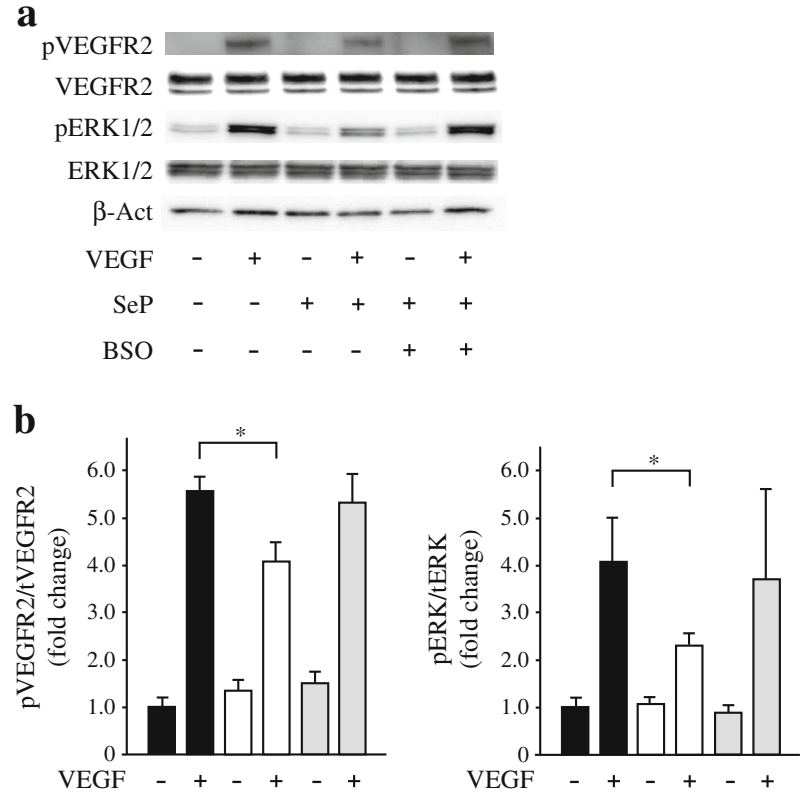

c

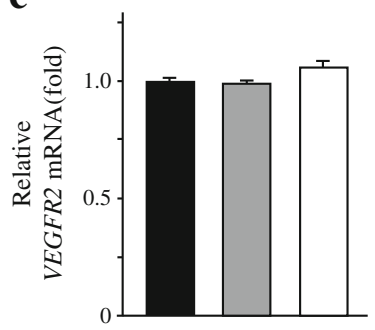

d

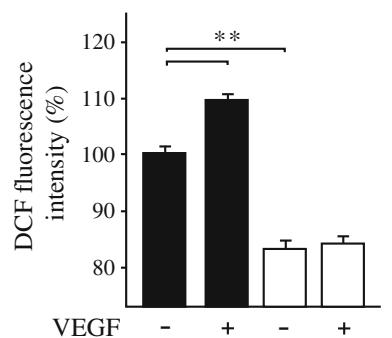

Fig. 3 SeP impairs VEGF signal transduction in endothelial cells. (a) VEGF signalling in HUVECs treated with SeP $(10 \mu \mathrm{g} / \mathrm{ml})$. (b) Quantification of phosphorylated VEGFR2 and ERK normalised to total VEGFR2 and total ERK in HUVECs $(n=6)$. (c) Gene expression levels for $V E G F R 2$ in HUVECs treated with $\mathrm{SeP}$ for $24 \mathrm{~h}$ normalised to GAPDH $(n=6)$. (d) ROS levels in HUVECs stimulated with VEGF for 5 min $(n=8)$. ROS levels were measured as DCF fluorescence intensity. Black bars, control; dark-grey bars, SeP $5 \mu \mathrm{g} / \mathrm{ml}$; white bars, SeP $10 \mu \mathrm{g} / \mathrm{ml}$; light-grey bars, SeP $10 \mu \mathrm{g} / \mathrm{ml}$ and BSO $0.2 \mathrm{mmol} / \mathrm{l}$. Data are mean \pm SEM. $* p<0.05$ and $* * p<0.01$. WT, wild-type
VEGF. The VEGF-induced ROS burst is reported to be required for the subsequent VEGF signal transduction [27]. Stimulation with $50 \mathrm{ng} / \mathrm{ml}$ VEGF for 5 min significantly increased intracellular levels of ROS in HUVECs (Fig. 3d). Pretreatment with SeP suppressed intracellular levels of ROS both with and without VEGF stimulation (Fig. 3d). These results suggest that SeP-induced VEGF resistance is associated with a reduction in the ROS burst stimulated by VEGF.

$\mathrm{SeP}$ delays wound healing of skin in mice To clarify whether hepatic overexpression of SeP affects angiogenesis-related disorder in vivo, we used a hydrodynamic injection method to generate mice that overexpress human SEPP $1 \mathrm{mRNA}$ in the liver. Levels of SEPP1 gene expression in the liver and SeP protein in the blood were significantly elevated in these mice (Fig. 4a, b), whereas serum levels of total selenium in wildtype and SeP-transgenic mice, which were $322.6 \mathrm{ng} / \mathrm{ml}$ and $331.0 \mathrm{ng} / \mathrm{ml}$ respectively, were not significantly different (Fig. 4c).

We created excisional wounds $(10 \mathrm{~mm})$ in the dorsal skin of the mice and quantified the rate of wound healing. Wound closure was significantly impaired in the mice overexpressing SEPP1 at 3, 5 and 7 days (Fig. 4d, e). In contrast, Sepp $1^{-/-}$mice showed an improvement of the wound closure at 9 days compared with the wild-type animals (Fig. 4f, g). These results indicate that the hepatokine SeP delays the wound healing of the skin in mice.

Sepp1-heterozygous-knockout mice show enhanced angiogenesis after hindlimb ischaemia To determine whether attenuation of SeP expression enhances angiogenesis in vivo, we generated hindlimb ischaemia in Sepp $1^{+-}$mice. We previously reported that Sepp 1 -homozygous-knockout mice exhibit enhancement of insulin signalling in skeletal muscle, whereas Seppl-heterozygous-knockout mice show marginal changes in insulin signalling [15]. Hence, we selected Sepp 1-heterozygous-knockout mice in the present study to assess the direct actions of $\mathrm{SeP}$ on the vascular system, independent of insulin signalling. At 5 days after femoral artery ligation, Sepp $1^{+/}$mice showed a significant increase in blood flow compared with wild-type mice (Fig. 5a). This increase continued for 15 days after artery ligation (Fig. 5b). Consistent with these findings, histological examination showed increased vessel density in the hindlimb musculature as determined by immunostaining with anti-CD31 antibody (Fig. 5c, d).

\section{Discussion}

The present study indicates that the liver-derived secretory protein SeP impairs angiogenesis both in vitro and in vivo. SeP directly attenuates VEGF signal transduction in vascular 


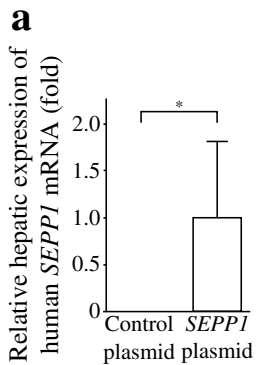

b

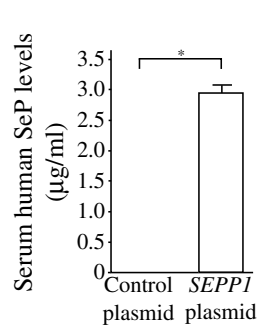

c

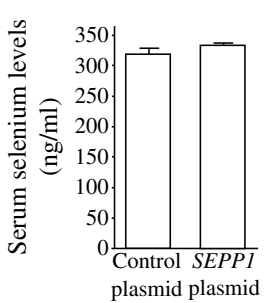

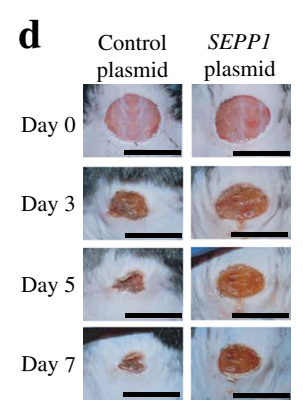

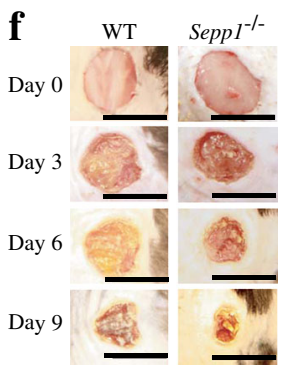

e

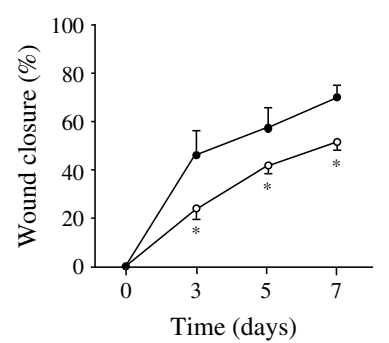

g

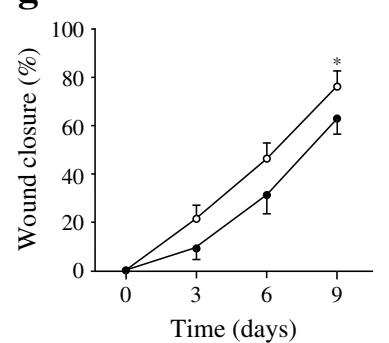

Fig. 4 Hepatic overexpression of SeP impairs wound healing in mice. (a) Levels of human SEPP1 mRNA normalised to $18 \mathrm{~S}$ rRNA in the livers of mice injected with plasmid DNA via the tail vein $(n=9)$. (b) Serum human SeP levels in mice injected with a plasmid encoding SEPPI $(n=9)$. (c) Serum levels of selenium in mice injected with a plasmid encoding SEPP1 $(n=3)$. (d) Representative images of full-thickness excision rounds on the backs of mice injected with SEPP1 plasmid. (e) Quantification of wound closure in mice injected with SEPP1 plasmid (white circles) and control (black circles) $(n=9)$. (f) Representative images of full-thickness excisional wounds on the backs of Sepp $1^{-/}$mice. (g) Quantification of wound closure in Sepp ${ }^{-/}$mice (white circles) and control (black circles) $(n=6-12)$. Data are mean \pm SEM. $* p<0.05$, scale bars, $10 \mathrm{~mm}$. WT, wild-type

endothelial cells, resulting in suppression of VEGF-induced cell proliferation, migration and tube formation. We reported previously that levels of both hepatic SEPP1 mRNA and serum SeP protein are elevated in type 2 diabetes [15]. Taken together with our previous report, the present study suggests that hepatic overproduction of SeP may contribute to the onset of impaired angiogenesis in type 2 diabetes (Fig. 6).

The attenuated VEGF signal transduction, VEGF resistance, has been postulated as the molecular mechanism underlying the dysregulation of angiogenesis in people with type 2 diabetes [3, 11]. Waltenberger et al reported that circulating monocytes show attenuation of VEGF-induced chemotaxis in people with diabetes mellitus [28] and that VEGF-stimulated

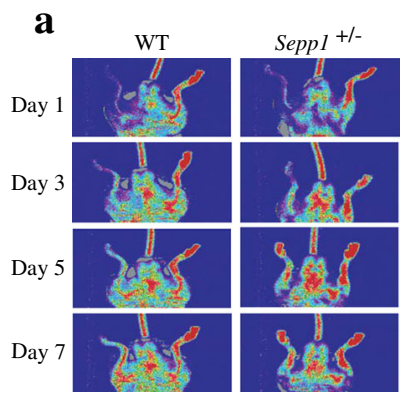

b
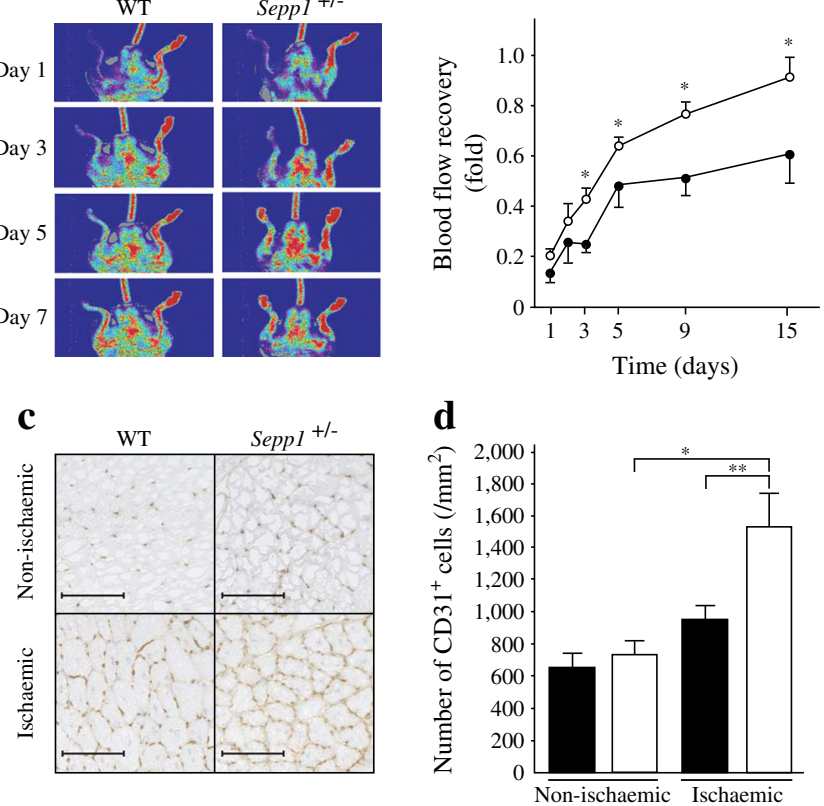

Fig. 5 Sepp $1^{+/-}$mice show enhanced angiogenesis during hindlimb ischaemia. (a) Representative images of perfusion recovery following hindlimb ischaemia in Sepp $1^{+/}$mice. (b) Quantification of blood flow recovery in Sepp $1^{+/-}$mice (white circles) and control (black circles) $(n=5)$. Ratios of perfusion from non-ischaemic leg to ischaemic leg are shown. (c) Representative images of CD31-stained sections of lower limb tissues of Sepp $1^{+/-}$mice at 15 days after ligation. Scale bars $100 \mu \mathrm{m}$. (d) Quantification of CD31-positive cells in the hindlimb of Sepp1 ${ }^{+/-}$mice (white bars) and WT (black bars). Data are from 16 fields per section. Data are mean \pm SEM. ${ }^{*} p<0.05$ and ${ }^{* *} p<0.01$

phosphorylation of downstream molecules is reduced in monocytes from patients with type 2 diabetes [29]. In addition, Sasso et al found impaired VEGF signalling in the myocardium of patients with type 2 diabetes and coronary

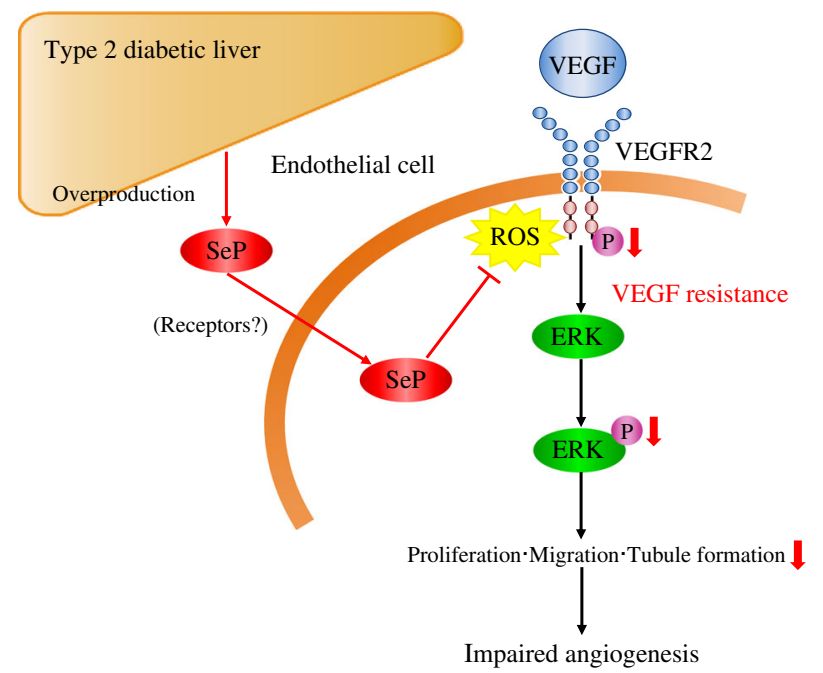

Fig. 6 Overproduction of SeP in type 2 diabetic liver induces VEGF resistance in vascular endothelial cells. SeP inhibits VEGF signal transduction by suppressing acute generation of ROS, resulting in the onset of impaired angiogenesis 
heart disease [30], suggesting that diabetes induces VEGF resistance in not only monocytes but also other types of cells such as cardiomyocytes and endothelial cells. However, the molecular mechanisms by which VEGF resistance arises in diabetes mellitus have not been elucidated. The results of the present study suggest a novel molecular pathology of type 2 diabetes; elevation of circulating SeP induces VEGF resistance in vascular endothelial cells.

The liver is the production site of various secretory proteins. Recent work in our laboratory has indicated that genes encoding secretory proteins are abundantly expressed in the liver in type 2 diabetes [31]. Moreover, genes encoding angiogenic factors, fibrogenic factors and redox-associated factors are differentially expressed in the liver in type 2 diabetes, possibly contributing to the pathophysiology and clinical manifestations of this disease $[32,33]$. The present study sheds light on a previously under-explored function of the liver; the liver may participate in the regulation of systemic angiogenesis by altering the production of angiogenesisassociated hepatokines such as SeP.

Our observation that $\mathrm{SeP}$ impairs angiogenic processes is noteworthy in the context of experimental data suggesting that SeP plays a role in the antioxidative defence system [13]. In fact, we have shown previously that $\mathrm{SeP}$ increases the activity of glutathione peroxidase 1 (GPX1), a representative antioxidative enzyme that requires selenium for its enzymatic action, in Jurkat E6-1 cells, a human T cell leukaemia cell line [14]. SeP-induced activation of GPX1 was also demonstrated in endothelial cells [19]. Accumulating evidence indicates that ROS stimulate the angiogenic response in order to initiate the tissue repair process in ischaemia-reperfusion lesions [34]. Among the growth factors involved in angiogenesis, VEGF plays a role in a ROS-dependent signal transduction system [27]. VEGF binding to VEGFR2 stimulates NADPH oxidase in endothelial cells, resulting in the acute generation of ROS such as hydrogen peroxide. This ROS burst oxidises and inactivates protein tyrosine phosphatases, which negatively regulate VEGF signalling and thereby promote VEGFR2 phosphorylation and the subsequent signalling cascade [27].

In combination with these previous reports, the present data suggest that SeP induces VEGF resistance in endothelial cells by increasing GPX1 and subsequently suppressing the VEGFinduced ROS generation that is required for VEGF signal transduction. This speculation was supported by our findings that the co-administration of BSO, an inhibitor of glutathione synthesis, rescued the inhibitory effects of SeP on VEGF signalling and the subsequent VEGF responsiveness. The identification of SeP receptor(s) in endothelial cells would provide further insight into the molecular mechanism by which SeP impairs VEGF signal transduction.

VEGF signalling is known to play paradoxical roles in the pathogenesis of diabetic complications. Both enhancement and suppression of angiogenesis are observed in different tissues in diabetic conditions [35]. In contrast to hindlimb ischaemia or wound healing, advanced diabetic retinopathy is characterised by VEGF-induced abnormal neovascularisation in the retina. Current management for diabetic retinopathy includes anti-VEGF therapy along with blood glucose control [36]. In addition to retinopathy, growing evidence indicates that VEGF-related abnormal angiogenesis plays a major role in diabetic nephropathy [37]. Moreover, a recent report showed that pharmacological inhibition of VEGF-B improves glucose tolerance and insulin resistance in rodent models with type 2 diabetes [38]. Additional studies are needed to determine the actions of $\mathrm{SeP}$ on the enhanced angiogenesis in diabetic retinopathy or nephropathy.

Unlike phosphorylation of VEGFR2 and ERK1/2, VEGFinduced phosphorylation of Akt, p38 MAPK and protein kinase, AMP-activated, $\alpha 1$ catalytic subunit (AMPK) was unchanged by SeP in HUVECs (data not shown). Although the detailed molecular mechanism by which SeP selectively impairs VEGFR2/ERK pathway in HUVECs is still unknown, SeP might act on ERK-selective MAPK phosphatases [39]. In fact, some MAPK phosphatases are inactivated by intracellular oxidative stress [39]. However, SeP-induced selective impairment of VEGFR2/ERK pathway should be confirmed in other vascular endothelial cells.

All the culture media we used for HUVECs in this study contained $5.5 \mathrm{mmol} / \mathrm{l}$ glucose, which corresponds to fasting plasma glucose levels in people with normal glucose tolerance. However, we confirmed that SeP also attenuated VEGF signalling of HUVECs in the presence of $25 \mathrm{mmol} / \mathrm{l}$ glucose (data not shown). These results suggest that $\mathrm{SeP}$ induces VEGF resistance in HUVECs in both normoglycaemic and hyperglycaemic conditions. However, additional experiments are clearly needed to determine whether SeP sufficiently removes hyperglycaemia-induced chronic oxidative stress in vascular endothelial cells.

We have shown that serum levels of total selenium were unchanged in the mice injected with SEPP1 plasmid compared with the control animals (Fig. 4c), in spite of the significant elevation of serum SeP (Fig. 4b). Selenium content in forms other than $\mathrm{SeP}$ might decrease in the serum of the SeP-transgenic mice compensatively $[14,40]$. Because a recent report showed that $\mathrm{SeP}$ exerts antioxidative actions independently of selenium supply [41], we speculate that the phenotype of the SeP-transgenic mice reflects the action of $\mathrm{SeP}$ itself, not the abnormal selenium distribution in mice.

Sepp1-heterozygous-knockout mice exhibited an increase in angiogenesis during hindlimb ischaemia without the induction of diabetes (Fig. 5), suggesting that the hepatokine SeP plays a role in the regulation of systemic angiogenesis, irrespective of diabetes status. For example, lipopolysaccharideinduced acute inflammation was reported to downregulate the production of $\mathrm{SeP}$ in mice [42]. Angiogenesis promoted by suppressed production of SeP might be beneficial in 
inflammatory conditions. Further characterisation of Sepp1deficient mice will provide insights into the involvement of $\mathrm{SeP}$ in the regulation of angiogenesis in normoglycaemic conditions.

Serum levels of human SeP in the mice injected with human SEPP1 plasmid reached approximately $2.0 \mu \mathrm{g} / \mathrm{ml}$ (Fig. 4b). This corresponds with the incremental change in serum level of SeP from that of people with normal glucose tolerance to that of people with type 2 diabetes in the Japanese population $[15,25]$. This strongly suggests that the phenotype observed in the SeP-transgenic mice reflects the physiological actions of SeP.

One limitation of the present study is that we examined the action of SeP on endothelial cells only. Various types of cell participate in the angiogenic processes. Further studies are necessary to determine whether SeP exerts effects on other cell types such as the monocytes or endothelial progenitor cells.

Another limitation of the present study is that we carried out all the experiments of Sepp1-deficient mice without the induction of diabetes with a high-fat diet or streptozotocin. Hence, we did not investigate the contribution of SeP in the development of the dysregulated angiogenesis seen in diabetes in vivo. However, our data indicate that treatment with purified SeP directly inhibits angiogenesis in both vascular endothelial cells and mice under euglycaemic conditions. Combined with the previous reports showing the elevated production of SeP in type 2 diabetes $[15,16]$, the current data suggest that overproduction of $\mathrm{SeP}$ contributes to the onset of impaired angiogenesis in type 2 diabetes. However, further studies in animals with diabetes are necessary to determine the degree of the contribution of $\mathrm{SeP}$ on the impaired angiogenesis observed in diabetes.

In summary, the present study indicates that the diabetesassociated hepatokine SeP impairs angiogenesis by reducing VEGF signal transduction in endothelial cells, and suggests that SeP may be a novel therapeutic target for treatment of VEGF resistance in people with type 2 diabetes.

Acknowledgements We thank M. Wakabayashi, Y. Furuta and Y. Hashimoto of Kanazawa University for technical assistance. We are indebted to K. E. Hill and R. F. Burk of Vanderbilt University School of Medicine for the Sepp1-knockout mice.

Some of the data were presented as an abstract at the 9th International Diabetes Federation Western Pacific Region Congress, 4th Scientific Meeting of the Asian Association for the Study of Diabetes, 24-27 November 2012, Kyoto, Japan, and at the 49th annual meeting of the European Association for the Study of Diabetes, 23-27 September 2013, Barcelona, Spain.

Funding This work was supported by Grants-in-Aid from the Ministry of Education, Culture, Sports, Science and Technology, Japan.

Duality of interest The authors declare that there is no duality of interest associated with this manuscript.
Contribution statement KI researched the data and wrote the manuscript. HM conceived and designed the experiments, researched the data, contributed to the discussion, wrote the manuscript and reviewed and edited the manuscript. MK researched the data, contributed to the discussion and reviewed and edited the manuscript. HT, NM-N, NTaj, KC, FL, $\mathrm{HA}, \mathrm{TO}, \mathrm{MS}, \mathrm{YT}, \mathrm{KK}, \mathrm{AF}$ and $\mathrm{KM}$ designed the experiments, contributed to the discussion and reviewed the manuscript. YS, YO, YT, KT, HK, SKam and NTak conceived and designed the experiments, researched the data, contributed to the discussion and revised the manuscript critically for important intellectual content. SKan and TT conceived and designed the experiments, contributed to the discussion, wrote the manuscript and reviewed and edited manuscript. TT is the guarantor of this work, has full access to all the data in the study and takes responsibility for the integrity of the data and accuracy of the data analysis. All the authors have approved the final version of the manuscript.

\section{References}

1. Holman RR, Paul SK, Bethel MA, Matthews DR, Neil HA (2008) 10-year follow-up of intensive glucose control in type 2 diabetes. N Engl J Med 359:1577-1589

2. Gerstein HC, Miller ME, Byington RP et al (2008) Effects of intensive glucose lowering in type 2 diabetes. $\mathrm{N}$ Engl $\mathrm{J}$ Med $358: 2545-2559$

3. Simons M (2005) Angiogenesis, arteriogenesis, and diabetes: paradigm reassessed? J Am Coll Cardiol 46:835-837

4. Abaci A, Oguzhan A, Kahraman S et al (1999) Effect of diabetes mellitus on formation of coronary collateral vessels. Circulation 99:2239-2242

5. Yarom R, Zirkin H, Stammler G, Rose AG (1992) Human coronary microvessels in diabetes and ischaemia. Morphometric study of autopsy material. J Pathol 166:265-270

6. Al-Delaimy WK, Merchant AT, Rimm EB, Willett WC, Stampfer MJ, Hu FB (2004) Effect of type 2 diabetes and its duration on the risk of peripheral arterial disease among men. Am J Med 116:236-240

7. Hueb W, Gersh BJ, Costa F et al (2007) Impact of diabetes on fiveyear outcomes of patients with multivessel coronary artery disease. Ann Thorac Surg 83:93-99

8. Galiano RD, Tepper OM, Pelo CR et al (2004) Topical vascular endothelial growth factor accelerates diabetic wound healing through increased angiogenesis and by mobilizing and recruiting bone marrow-derived cells. Am J Pathol 164:1935-1947

9. Boodhwani M, Sellke FW (2009) Therapeutic angiogenesis in diabetes and hypercholesterolemia: influence of oxidative stress. Antioxid Redox Signal 11:1945-1959

10. Jude EB, Eleftheriadou I, Tentolouris N (2010) Peripheral arterial disease in diabetes-a review. Diabet Med 27:4-14

11. Waltenberger $J$ (2009) VEGF resistance as a molecular basis to explain the angiogenesis paradox in diabetes mellitus. Biochem Soc Trans 37:1167-1170

12. Carlson BA, Novoselov SV, Kumaraswamy E et al (2004) Specific excision of the selenocysteine tRNA[Ser]Sec (Trsp) gene in mouse liver demonstrates an essential role of selenoproteins in liver function. J Biol Chem 279:8011-8017

13. Burk RF, Hill KE (2005) Selenoprotein P: an extracellular protein with unique physical characteristics and a role in selenium homeostasis. Annu Rev Nutr 25:215-235

14. Saito Y, Takahashi K (2002) Characterization of selenoprotein $P$ as a selenium supply protein. Eur J Biochem/FEBS 269:5746-5751

15. Misu H, Takamura T, Takayama $\mathrm{H}$ et al (2010) A liver-derived secretory protein, selenoprotein P, causes insulin resistance. Cell Metab $12: 483-495$ 
16. Yang SJ, Hwang SY, Choi HY et al (2011) Serum selenoprotein P levels in patients with type 2 diabetes and prediabetes: implications for insulin resistance, inflammation, and atherosclerosis. J Clin Endocrinol Metab 96:E1325-E1329

17. Arteel GE, Franken S, Kappler J, Sies H (2000) Binding of selenoprotein $\mathrm{P}$ to heparin: characterization with surface plasmon resonance. Biol Chem 381:265-268

18. Burk RF, Hill KE, Boeglin ME, Ebner FF, Chittum HS (1997) Selenoprotein $\mathrm{P}$ associates with endothelial cells in rat tissues. Histochem Cell Biol 108:11-15

19. Steinbrenner H, Bilgic E, Alili L, Sies H, Brenneisen P (2006) Selenoprotein $\mathrm{P}$ protects endothelial cells from oxidative damage by stimulation of glutathione peroxidase expression and activity. Free Radic Res 40:936-943

20. Takayama H, Misu H, Iwama H et al (2013) Metformin suppresses expression of the selenoprotein $\mathrm{P}$ gene via an AMPK-FoxO3a pathway in H4IIEC3 hepatocytes. J Biol Chem

21. Hill KE, Zhou J, McMahan WJ et al (2003) Deletion of selenoprotein $\mathrm{P}$ alters distribution of selenium in the mouse. J Biol Chem 278:13640-13646

22. Watkinson JH (1966) Fluorometric determination of selenium in biological material with 2,3-diaminonaphthalene. Anal Chem 38:92-97

23. Abdulah R, Miyazaki K, Nakazawa M, Koyama H (2005) Low contribution of rice and vegetables to the daily intake of selenium in Japan. Int J Food Sci Nutr 56:463-471

24. Saito Y, Hayashi T, Tanaka A et al (1999) Selenoprotein P in human plasma as an extracellular phospholipid hydroperoxide glutathione peroxidase. Isolation and enzymatic characterization of human selenoprotein p. J Biol Chem 274:2866-2871

25. Saito Y, Watanabe Y, Saito E, Honjoh T, Takahashi K (2001) Production and application of monoclonal antibodies to human selenoprotein P. J Health Sci 47:346-352

26. Luttun A, Tjwa M, Moons L et al (2002) Revascularization of ischemic tissues by PlGF treatment, and inhibition of tumor angiogenesis, arthritis and atherosclerosis by anti-Flt1. Nat Med 8:831-840

27. Ushio-Fukai M (2007) VEGF signaling through NADPH oxidasederived ROS. Antioxid Redox Signal 9:731-739

28. Waltenberger J, Lange J, Kranz A (2000) Vascular endothelial growth factor-A-induced chemotaxis of monocytes is attenuated in patients with diabetes mellitus: a potential predictor for the individual capacity to develop collaterals. Circulation 102:185-190

29. Tchaikovski V, Olieslagers S, Bohmer FD, Waltenberger J (2009) Diabetes mellitus activates signal transduction pathways resulting in vascular endothelial growth factor resistance of human monocytes. Circulation 120:150-159

30. Sasso FC, Torella D, Carbonara O et al (2005) Increased vascular endothelial growth factor expression but impaired vascular endothelial growth factor receptor signaling in the myocardium of type 2 diabetic patients with chronic coronary heart disease. J Am Coll Cardiol 46:827-834

31. Misu H, Takamura T, Matsuzawa N et al (2007) Genes involved in oxidative phosphorylation are coordinately upregulated with fasting hyperglycaemia in livers of patients with type 2 diabetes. Diabetologia 50:268-277

32. Takamura T, Sakurai M, Ota T, Ando H, Honda M, Kaneko S (2004) Genes for systemic vascular complications are differentially expressed in the livers of type 2 diabetic patients. Diabetologia 47:638-647

33. Takamura T, Misu H, Matsuzawa-Nagata N et al (2008) Obesity upregulates genes involved in oxidative phosphorylation in livers of diabetic patients. Obesity 16:2601-2609

34. Maulik N, Das DK (2002) Redox signaling in vascular angiogenesis. Free Radic Biol Med 33:1047-1060

35. Costa PZ, Soares R (2013) Neovascularization in diabetes and its complications. Unraveling the angiogenic paradox. Life Sci 92:1037-1045

36. Gupta N, Mansoor S, Sharma A et al (2013) Diabetic retinopathy and VEGF. Open Ophthalmol J 7:4-10

37. Nakagawa T, Kosugi T, Haneda M, Rivard CJ, Long DA (2009) Abnormal angiogenesis in diabetic nephropathy. Diabetes 58:1471-1478

38. Hagberg CE, Mehlem A, Falkevall A et al (2012) Targeting VEGF-B as a novel treatment for insulin resistance and type 2 diabetes. Nature 490:426- 430

39. Patterson KI, Brummer T, O'Brien PM, Daly RJ (2009) Dualspecificity phosphatases: critical regulators with diverse cellular targets. Biochem J 418:475-489

40. Deagen JT, Beilstein MA, Whanger PD (1991) Chemical forms of selenium in selenium containing proteins from human plasma. J Inorg Biochem 41:261-268

41. Kurokawa S, Eriksson S, Rose KL et al (2014) Sepp1(UF) forms are $\mathrm{N}$-terminal selenoprotein $\mathrm{P}$ truncations that have peroxidase activity when coupled with thioredoxin reductase-1. Free Radic Biol Med 69:67-76

42. Renko K, Hofmann PJ, Stoedter M et al (2009) Down-regulation of the hepatic selenoprotein biosynthesis machinery impairs selenium metabolism during the acute phase response in mice. FASEB J 23: $1758-1765$ 